\title{
A Novel Drug Delivery Carrier Comprised of Nimodipine Drug Solution and a Nanoemulsion: Preparation, Characterization, in vitro, and in vivo Studies
}

This article was published in the following Dove Press journal:

International Journal of Nanomedicine

Saixu Huang, ${ }^{1-3, *}$

Zhiyong Huang, ${ }^{1-3, *}$

Zhiqin Fu, (D) ${ }^{3, *}$ Yamin Shi, ${ }^{3,4}$

Qi Dai, (D) ${ }^{1,2}$ Shuyan Tang, ${ }^{3}$

Yongwei $\mathrm{Gu},{ }^{3}$ Youfa $\mathrm{Xu}{ }^{3}$ Jianming Chen, ${ }^{3,4}$ Xin Wu, ${ }^{3}$ Fuzheng Ren (iD) ${ }^{1,2}$

'Shanghai Key Laboratory of New Drug Design, School of Pharmacy, East China University of Science and Technology, Shanghai, People's Republic of China; ${ }^{2}$ Engineering Research Centre of Pharmaceutical Process Chemistry, Ministry of Education, East China University of Science and Technology, Shanghai, People's Republic of China; ${ }^{3}$ Shanghai Weier Biological Medicine Science and Technology Co. Ltd., Shanghai, People's Republic of China; ${ }^{4}$ Department of Pharmacy, Fujian University of Traditional Chinese Medicine, Fujian, People's Republic of China

*These authors contributed equally to this work

Correspondence: Fuzheng Ren School of Pharmacy, East China University of Science and Technology, No. 130,

Meilong Road, Shanghai 200237, People's

Republic of China

Tel/Fax +86 2l 64253255

Email fzren@ecust.edu.cn

Xin Wu

Shanghai Weier Biological Medicine

Science and Technology Co. Ltd., No. 358

Tian Chen Road, Shanghai 201799 .

People's Republic of China

$\mathrm{Tel} / \mathrm{Fax}+862131198947$

Email xinwu007@I26.com
Purpose: Nimodipine (NIMO) is used clinically to treat ischemic damage resulting from subarachnoid hemorrhage. However, clinical application of NIMO is limited by poor aqueous solubility and low safety. To overcome these limitations, a novel two-vial NIMO-loaded nanoemulsion (NIMO-TNE) was designed in this study.

Methods: NIMO-TNE was prepared by mixing a nimodipine-polyethylene glycol 400 (NIMO-PEG400) solution and a commercially available $20 \%$ injectable blank nanoemulsion (BNE). Drug distribution in NIMO-TNE, physical stability, and dilution stability were evaluated in vitro, and pharmacokinetics and pharmacodynamics were evaluated in vivo. Safety was assessed using the hemolysis test and the intravenous irritation test, and acute toxicity of NIMO-TNE was compared with that of commercial Nimotop injection.

Results: Drug loading (DL) in NIMO-TNE was enhanced 5-fold compared with that in Nimotop injection. The mean particle size of NIMO-TNE was $241.53 \pm 1.48 \mathrm{~nm}$. NIMOTNE and NIMO-TNE diluted in 5\% glucose injection and $0.9 \%$ sodium chloride was stable for a sufficient duration to allow for clinical use. In addition, NIMO-TNE exhibited a similar pharmacokinetic profile and similar brain ischemia reduction in a rat middle cerebral artery occlusion (MCAO) model compared to Nimotop injection. Furthermore, NIMO-TNE did not induce hemolysis at $37^{\circ} \mathrm{C}$, and NIMO-TNE induced less intravenous irritation than Nimotop injection. Moreover, NIMO-TNE could be injected at a 23-fold higher dose than the $\mathrm{LD}_{50}$ of Nimotop injection with no obvious toxicity or side effects.

Conclusion: NIMO-TNE is a promising formulation suitable for intravenous injection, is easy to prepare, and exhibits excellent safety.

Keywords: nimodipine, nanoemulsion, pharmacokinetics, MCAO, LD50

\section{Introduction}

Nimodipine (NIMO), a dihydropyridine calcium antagonist, was originally developed by Bayer in 1982, and has been shown to dilate the cerebral arteries and increase cerebrovascular flow. ${ }^{1}$ Nimodipine is a preferred drug for treatment of ischemic cerebrovascular disease, and is also used to treat Alzheimer's disease, stroke, migraine,

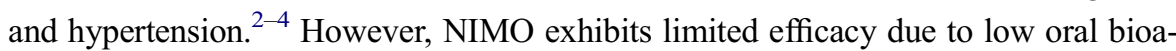
vailability and poor water solubility. ${ }^{5-7}$ Therefore, NIMO is administered intravenously, resulting in higher bioavailability than that observed following oral administration. Nimotop injection, produced by Bayer, is a commercially available 
injection that contains $23.7 \%(\mathrm{v} / \mathrm{v})$ ethanol and $17 \%(\mathrm{v} / \mathrm{v})$ polyethylene glycol 400 (PEG 400) to increase drug concentration in the formulation. ${ }^{8}$ The high concentration of organic solvent induces blood vessel irritation, resulting in pain and inflammation at the injection site. Furthermore, daily exposure to organic solvent is $101.7 \mathrm{~mL}$ according to the recommended NIMO dose, which may lead to phlebitis during infusion and poor compliance. ${ }^{6,9-13}$ In addition, drug crystallization may occur when Nimotop injection is diluted with glucose or saline solutions due to poor water solubility, which can result in increased patient health risk. Therefore, there is an urgent need to develop safe and effective injectable nimodipine formulations.

Nanoparticle drug delivery systems have been widely used for injection of poorly soluble drugs because they allow for high drug loading, good biocompatibility, and sustained release. ${ }^{14}$ Injectable nanoparticle formulations of NIMO have been developed previously, and include micelles, liposomes, and solid lipid nanoparticles. ${ }^{8,15-20}$ These nano-drug delivery systems have general advantages over Nimotop injection, but they typically exhibit poor stability, are subject to phagocytic effects in the reticuloendothelial system, and are difficult to produce. We initially designed a one-vial NIMO-loaded nanoemulsion, but this formulation exhibited poor stability. The drug content in the one-vial formulation decreased below $98 \%$ when stored at $25{ }^{\circ} \mathrm{C} \pm 2{ }^{\circ} \mathrm{C}$ with a relative humidity of $60 \% \pm 5 \%$ after only $72 \mathrm{hrs}$. To improve stability, a novel drug delivery carrier comprised of a commercially available injectable blank nanoemulsion (BNE) and a 4\% (v/v) organic drug solution was designed based on our previous study. ${ }^{21}$ These two parts were designed to be mixed prior to clinical use to form an injectable drug-loaded nanoemulsion. Separation of the components of the formulation until use resulted in significantly improved stability. In addition, the amount of organic solvent was significantly lower than that in Nimotop injection due to high drug loading, which resulted in improved safety and less side effects. This novel two-vial drug-loaded nanoemulsion formulation may be an ideal drug carrier for intravenous injection of NIMO.

The objective of this study was to prepare a novel twovial NIMO-loaded nanoemulsion (NIMO-TNE) that exhibited good safety and was easy to prepare. We evaluated drug distribution in NIMO-TNE, and physical and dilution stability of NIMO-TNE. Moreover, pharmacokinetic and pharmacodynamic properties of NIMO-TNE were compared with those of Nimotop injection in rats. Safety of NIMO-TNE was evaluated using the hemolysis test, assessment of intravenous irritation, and acute toxicity of NIMO-TNE was compared with that resulting from Nimotop injection.

\section{Materials and Methods Materials}

Nimodipine was purchased from Kangmanlin Chemical Industry Co., Ltd (Nanjing, China). Nitrendipine, used as an internal standard, was purchased from the National Institute of Food and Drug Control (Beijing, China). Twenty percent $(\mathrm{w} / \mathrm{w})$ aqueous injectable emulsion (longchain oil: medium-chain oil, 1:1, w/w) was purchased from Baxter Qiaoguang Healthcare Co., Ltd (Guangzhou, China). Nimotop injection $(10 \mathrm{mg} / 50 \mathrm{~mL})$ was purchased from Bayer Pharma AG. PEG400 was obtained from Well Chemical Co., Ltd (Nanjing, China). Five percent glucose solution injection and $0.9 \%$ sodium chloride injection were purchased from Tianrui Pharmaceutical Co., Ltd (Zhejiang, China). Two percent 2,3,5-triphenyl tetrazolium chloride, $4 \%$ paraformaldehyde solution, and $10 \%$ paraformaldehyde were purchased from Yuanye Biotechnology Co., Ltd (Shanghai, China). Heparin sodium salt, chloral hydrate, diethyl ether, and n-hexane were of AR grade and were purchased from Sinopharm Chemical Reagent Co., Ltd. Methanol (HPLC grade) and acetonitrile (HPLC grade) were purchased from Thermo Fisher (China) Co., Ltd. All other chemical reagents were of analytical grade or chromatographic grade.

\section{Animals}

Thirty-two male ICR mice (SPF, 20-25g), thirty-two female ICR mice (SPF, 20-25g), twelve male Sprague-Dawley rats (SPF, 250-300 g), twenty-four male Sprague-Dawley rats (SPF, 140-160g), and six male rabbits $(2.5-3.0 \mathrm{~kg}$ ) were purchased from The Second Military Medical University and were housed at a temperature of $25^{\circ} \mathrm{C} \pm 2{ }^{\circ} \mathrm{C}$ with relative humidity maintained at $47.5 \% \pm 2.5 \%$. All animal studies were performed in accordance with the Ethical Guidelines for Investigations in Laboratory Animals issued by the Second Military Medical university (Shanghai, People's Republic of China). The animals were allowed to acclimate for 3 days prior to experimentation. Animals were fed standard feed (SPF level, Liaoning Qianmin Feedstuff Co., Ltd.) and provided autoclaved purified water. The animals were subjected to a $12 \mathrm{~h}$ light/12 h dark cycle. Following the experiments, the animal carcasses were frozen then collected by professional companies. The protocols and 
procedures were approved by the Institutional Animal Care and Use Committee of the Second Military Medical university (Shanghai, People's Republic of China).

\section{Preparation of NIMO-TNE}

NIMO-TNE was prepared using NIMO-PEG400 solution and blank fat emulsion. The blank fat emulsion was a commercially available $20 \%$ injectable BNE. NIMOPEG400 was prepared by dissolving NIMO $(0.25 \mathrm{~g})$ in PEG400 (10 mL) using ultrasound to aid dissolution. The NIMO-PEG400 solution was then sterilized at $121^{\circ} \mathrm{C}$ for $15 \mathrm{~min}$. NIMO-TNE $(1 \mathrm{mg} / \mathrm{mL})$ was prepared by combining $4 \mathrm{~mL}$ of NIMO-PEG400 solution with $96 \mathrm{~mL}$ of BNE, then shaking 30 times by rotating the vessel 180 degrees (about 30 seconds) at $25{ }^{\circ} \mathrm{C} \pm 2{ }^{\circ} \mathrm{C}$ at a relative humidity of $60 \% \pm 5 \%$.

\section{Characterization of NIMO-TNE Analysis of NIMO Using HPLC}

High performance liquid chromatography (HPLC) analysis was performed for drug quantitation using an Agilent 1260II HPLC system comprised of an Agilent G7111A pump and an Agilent G7114A-VWD detector. Data acquisition and processing were performed using Agilent openlab2.3 software.

Separation was performed on an Agilent Eclipse Plus C18 column $(250 \times 4.6 \mathrm{~mm}, 5 \mu \mathrm{m})$ maintained at $30{ }^{\circ} \mathrm{C}$. The mobile phase consisted of methanol: acetonitrile: water $(35: 38: 27 \mathrm{v} / \mathrm{v} / \mathrm{v})$. The flow rate was $1.0 \mathrm{~mL} / \mathrm{min}$ and the injection volume was $10 \mu \mathrm{L}$. Absorbance was monitored at $235 \mathrm{~nm}$.

\section{Drug Distribution in NIMO-TNE}

The drug distribution in NIMO-TNE was estimated as previously described. ${ }^{7,21-24}$ Drug content in the aqueous phase was determined using ultrafiltration and centrifugation. An appropriate amount of NIMO-TNE $(4 \mathrm{~mL})$ was transferred to an ultrafiltration centrifuge tube $(100 \mathrm{k}$, Millipore Corporation, USA), and the tube was inserted into a $15-\mathrm{mL}$ centrifuge tube. After centrifuging at $7000 \mathrm{rpm}$ for $15 \mathrm{~min}$ at $4{ }^{\circ} \mathrm{C}$ (Kecheng Instrument Equipment Co., Ltd, Hunan, China), the aqueous phase was collected and analyzed using HPLC. The oil phase was separated by addition of inorganic salt. Three grams of sodium sulfate was added to $8 \mathrm{~mL}$ of NIMO-TNE and the resulting sample was placed in a $15-\mathrm{mL}$ centrifuge tube. Centrifugation at $12,000 \mathrm{rpm}$ for $90 \mathrm{~min}$ at $4{ }^{\circ} \mathrm{C}$ resulted in the following three layers from top to bottom: oil phase, phospholipid bilayer, and aqueous phase. The oil phase was placed in a $2-\mathrm{mL}$ centrifuge tube and centrifuged at $10,000 \mathrm{rpm}$ for $5 \mathrm{~min}$ at $4{ }^{\circ} \mathrm{C}$. Fifty microliters of the oil phase was collected and diluted with diethyl ethermethanol (1:9) to $10 \mathrm{~mL}$, and drug content was measured using HPLC. The drug content in the phospholipid layer (Cp) was calculated using the following formula:

$$
C_{p}=\frac{C_{t}-C_{a}-C_{o}}{C_{t}} \times 100 \%,
$$

where $\mathrm{Cp}$ was the drug content in the phospholipid layer, $\mathrm{Ct}$ was the total drug content in NIMO-TNE, $\mathrm{Ca}$ was the drug content in the aqueous phase, and Co was the drug content in the oil phase.

\section{Measurement of Particle Size and $\mathrm{pH}$}

The mean particle size and polydispersity index (PDI) of NIMO-TNE were measured using a Nano $S$ Zetasizer (Malvern Instruments Ltd., UK) at $25{ }^{\circ} \mathrm{C}$. The samples were analyzed following $20 \mathrm{X}$ dilution with double-distilled water. The $\mathrm{pH}$ values of NIMO-TNE were measured using a PB-10 pH detector (Sartorius Ltd., Germany).

\section{Physical Stability Study}

NIMO-TNE $(1.0 \mathrm{mg} / \mathrm{mL}$ drug loading) was freshly prepared and stored at $25{ }^{\circ} \mathrm{C} \pm 2{ }^{\circ} \mathrm{C}$ at a relative humidity of $60 \% \pm$ $5 \%{ }^{21}$ Samples were analyzed for $\mathrm{pH}$, drug content, particle size, and PDI at predetermined time intervals. Long-term stability of NIMO-PEG400 solution $(25 \mathrm{mg} / \mathrm{mL})$ was evaluated under the same experimental conditions for 12 months. ${ }^{21}$

\section{Dilution Stability Study}

NIMO-TNE was diluted to the same concentration as Nimotop injection $(0.04 \mathrm{mg} / \mathrm{mL}$ in $5 \%$ glucose or $0.9 \%$ sodium chloride injection). ${ }^{25}$ The diluted samples were stored at $25{ }^{\circ} \mathrm{C} \pm 2{ }^{\circ} \mathrm{C}$ at a relative humidity of $60 \% \pm$ $5 \%$. The samples were analyzed at the predetermined time intervals for drug content using HPLC. Drug content, $\mathrm{pH}$, particle size, and PDI were evaluated to determine dilution stability.

\section{In vivo Pharmacokinetic Study}

\section{Groups and Dosing}

Twelve male Sprague-Dawley rats (250-300 g) were randomly divided into two groups with six rats in each group. NIMO-TNE was diluted with $0.9 \%$ sodium chloride to the same concentration as Nimotop injection. NIMO-TNE and Nimotop were injected intravenously via the tail vein at 
a single dose of $0.8 \mathrm{mg} / \mathrm{kg}$. Blood samples $(0.5 \mathrm{~mL})$ were collected from the ocular vein at $2,5,10,15,30,45,60$, 120 , and 240 min following administration. Plasma samples were immediately centrifuged at $4000 \mathrm{rpm}$ for $15 \mathrm{~min}$ at $4{ }^{\circ} \mathrm{C}$. Two hundred microliters of plasma was collected and immediately stored at $-20{ }^{\circ} \mathrm{C}$ until further analysis.

\section{Sample Preparation}

Plasma drug concentration was determined as follows. Forty microliters of internal standard $(5 \mu \mathrm{g} / \mathrm{mL}$ nitrendipine in methanol) was added to each $200 \mu \mathrm{L}$ plasma sample, and the samples were vortex-mixed for $30 \mathrm{~s}$. Then, $100 \mu \mathrm{L}$ of $\mathrm{NaOH}(1 \mathrm{~mol} / \mathrm{L})$ and $1.5 \mathrm{~mL}$ of $\mathrm{N}$-hexane: ethyl ether $(1: 1)$ were added, and the mixture was vortexed for $5 \mathrm{~min}$ and sonicated for $5 \mathrm{~min}$ at room temperature. ${ }^{8,19,26}$ The mixture was then centrifuged at $10,000 \mathrm{rpm}$ for $10 \mathrm{~min}$ at $4{ }^{\circ} \mathrm{C}$, the supernatant was transferred to a $1.5-\mathrm{mL}$ centrifuge tube, and the sample was evaporated using a RVC2-18CD plus vacuum desiccator (Christ Company, Germany) at $45{ }^{\circ} \mathrm{C}$. The dried residue was dissolved in $100 \mu \mathrm{L}$ of mobile phase, then centrifuged at $4000 \mathrm{rpm}$ for $15 \mathrm{mins}$ at $4{ }^{\circ} \mathrm{C}$. Twenty microliters of the supernatant was injected for HPLC analysis.

\section{Analysis of NIMO Using HPLC}

Plasma samples were separated using an Agilent Eclipse Plus $\mathrm{C} 18$ column $(250 \times 4.6 \mathrm{~mm}, 5 \mu \mathrm{m})$ maintained at $30^{\circ}$ C. The mobile phase was a mixture of acetonitrile water $(60: 40, \mathrm{v} / \mathrm{v})$, and the flow rate was $1.0 \mathrm{~mL} / \mathrm{min}^{8}$ Absorbance was monitored at $358 \mathrm{~nm}$.

\section{In vivo Pharmacodynamic Study Groups and Dosing}

Twenty-four Male Sprague-Dawley rats (140-160 g) were divided into the following three groups using a table of randomized numbers: Normal saline (NS) group $(\mathrm{n}=8)$; Nimotop injection group $(\mathrm{n}=8)$; and NIMO-TNE group $(\mathrm{n}=8)$. NIMO-TNE was diluted with $0.9 \%$ sodium chloride to the same concentration as Nimotop injection. NIMOTNE and Nimotop were injected at a single dose of $0.8 \mathrm{mg} / \mathrm{kg}$. Equal volumes of normal saline, Nimotop, and NIMO-TNE were injected intravenously via the tail vein.

\section{Middle Cerebral Artery Occlusion (MCAO) Model}

Electrocoagulation was used to generate a rat middle cerebral artery occlusion (MCAO) model as previously described. ${ }^{27-30}$ Sprague-Dawley rats were anesthetized by intraperitoneal injection with $3 \%$ pentobarbital sodium at a dose of $30 \mathrm{mg} / \mathrm{kg}$. The rats were placed in the lateral position on the operating table, and the left side of the face was shaved. An incision was made in the skin between the left orbit and the left ear following disinfection using $75 \%$ ethanol. The diaphragm and masseter muscle were separated and the tibia flap was exposed. A $2 \mathrm{~mm} \times 2 \mathrm{~mm}$ hole was made in the bone using a Strong 102L skull drill (Precision Instrument Factory, Korea), and the skull was opened under an XTS-4A microscope (Zhongtian Optical Instrument Co., Ltd, Jiangsu, China). The middle cerebral artery was electrocauterized $1 \mathrm{~mm}$ below the intersection with the olfactory tract using a bipolar electrocautery (Jinbaiwei Photoelectric Technology Co., Ltd, Wuhan, China). The soft tissue was repositioned, and the skin was sutured. The rats were then dosed with the control solution or the drug products, then placed in their cages with enough food and water for $24 \mathrm{~h}$.

The rats were anesthetized intraperitoneally with $3 \%$ pentobarbital sodium at a dose of $30 \mathrm{mg} / \mathrm{kg} 24 \mathrm{~h}$ after the MCAO procedure. The brains were immediately removed and sliced into 1-mm-thick coronal sections (Yuyan Scientific Instrument Co., Ltd, Shanghai, China). The slices were stained with a $1 \%$ solution of 2,3,5- triphenyl tetrazolium at $37{ }^{\circ} \mathrm{C}$ for $10 \mathrm{~min}$. Viable brain tissue was stained red, and the infarcted area was unstained and appeared white. The slices were then transferred to a $10 \%$ formaldehyde solution and stored in the dark for $24 \mathrm{~h}$ for fixation. Images of the slices were acquired using a digital camera (Figure 1), and the infarcted area was measured using image analysis software (Image J, version: 1.4.3.67). The ratio of infarct volumes was obtained by

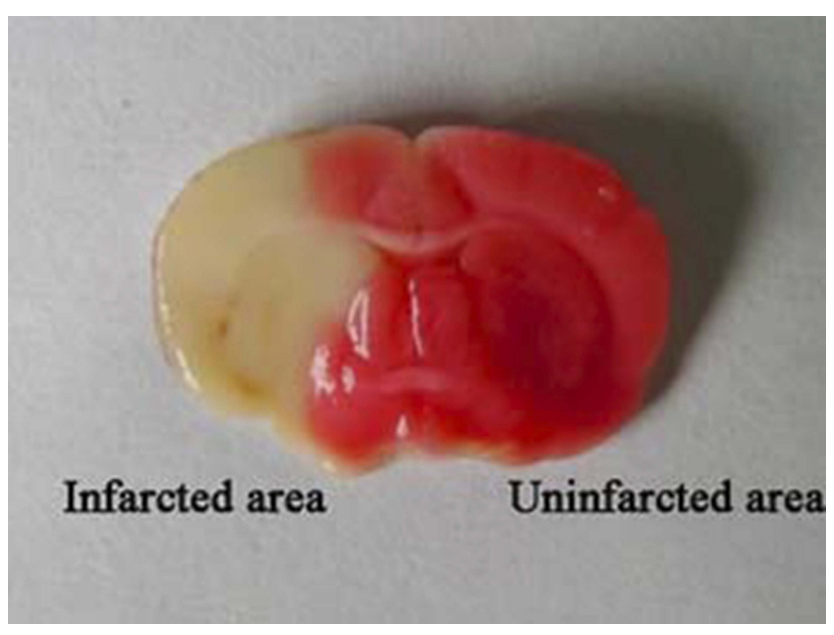

Figure I Stained coronal sections of rat brain following MCAO. Abbreviation: MCAO, Middle cerebral artery occlusion. 
calculating the percentage of infarct size compared to the total area of the infarcted cerebral hemisphere. ${ }^{28,30-32}$

\section{Safety Assessment Hemolysis Test}

The hemolysis test was used to evaluate the safety of NIMO-TNE for clinical use. Fresh rabbit blood $(10 \mathrm{~mL})$ was collected, and the fibrinogen fraction was removed by stirring with a glass rod. The erythrocytes in the defibrinated blood were washed three times with $10 \mathrm{~mL}$ of $0.9 \%$ saline solution. Each wash step included centrifugation at $2500 \mathrm{rpm}$ for $5 \mathrm{~min}$, followed by removal of the supernatant. A $2 \%(\mathrm{v} / \mathrm{v})$ erythrocyte suspension was prepared by diluting the erythrocytes with $0.9 \%$ saline solution.

Nine glass test tubes containing $2.5 \mathrm{~mL}$ of $2 \%(\mathrm{v} / \mathrm{v})$ erythrocyte suspension were labeled numerically. Various amounts of NIMO-TNE $(0.1,0.2,0.3,0.4$, and $0.5 \mathrm{~mL})$ were added to tubes $1-5$. Tube 6 contained only $2.5 \mathrm{~mL}$ of $0.9 \%$ saline, and served as a negative control. Tube 7 contained $2.5 \mathrm{~mL}$ of distilled water and served as a positive control. Tube 8 contained $2.5 \mathrm{~mL}$ of Nimotop injection, and served as a reference control. Blank nanoemulsion $(0.5 \mathrm{~mL})$ was added to tube 9 . Saline solution was added to each tube to a final volume of $5 \mathrm{~mL}$. The samples were incubated at $37{ }^{\circ} \mathrm{C}$ following mixing, and were observed at $15 \mathrm{~min}, 30 \mathrm{~min}, 45 \mathrm{~min}, 1 \mathrm{~h}, 2 \mathrm{~h}, 3 \mathrm{~h}$, and $4 \mathrm{~h}^{33,34}$

\section{Intravenous Irritation Evaluation}

Six male rabbits weighing $2.5-3.0 \mathrm{~kg}$ were randomly divided into two groups that received either Nimotop injection or NIMO-TNE. Nimotop injection and NIMOTNE were administered at a dose of $0.4 \mathrm{mg} / \mathrm{kg}$ via the marginal vein of the right ear, and an equivalent volume of $0.9 \%$ saline was injected via the marginal vein of the left ear. ${ }^{8,34,35}$ All injections were administered at a rate of $1 \mathrm{~mL} / \mathrm{min}$ once per day for three consecutive days. The appearance of the injection sites and the surrounding tissue was visually observed during the experiment. All rabbits in the study were euthanized $24 \mathrm{~h}$ after the final injection, and tissue samples located $0.5 \mathrm{~cm}$ and $3 \mathrm{~cm}$ from the injection site were cut. The tissue samples were fixed in $10 \%$ formalin, dehydrated using an ethanol gradient, embedded in paraffin, and stained with hematoxylin and eosin. All samples were examined using a BX43-DP21 light microscope (Olympus ltd. Japan) and pathological changes were evaluated.

\section{Acute Toxicity}

The median lethal dose $\left(\mathrm{LD}_{50}\right)$ of Nimotop injection was evaluated. The maximal feasible dose (MFD) of NIMOTNE was evaluated to assess acute toxicity, based on excellent safety observed in preliminary studies.

The $\mathrm{LD}_{50}$ of Nimotop injection was determined as follows. ${ }^{36-38}$ Forty ICR mice (20-25 g) were assigned randomly to five groups, with 4 males and 4 females in each group. Nimotop was injected intravenously via the tail vein at single doses of $1.90,2.10,2.32,2.56$, or $2.83 \mathrm{mg} / \mathrm{kg}$. The mice were observed for general behavior and signs of toxicity for $14 \mathrm{~d}$.

Twenty-four ICR mice (20-25 g) were divided randomly into a NIMO-TNE group and a control group comprised of 6 males and 6 females in each group to evaluate the maximum feasible dose of NIMO-TNE. Mice in the NIMO-TNE group were injected with NIMO-TNE at a dose of $18 \mathrm{mg} / \mathrm{kg}$ via the tail vein three times in a 24 $\mathrm{h}$ period $(54 \mathrm{mg} / \mathrm{kg}$ cumulative dose). The mice in the control group were not subjected to any experimental procedures. The mice were observed for general behavior and signs of toxicity for $14 \mathrm{~d}$. Changes in body weight were also monitored.

\section{Statistical Analysis}

All the results are presented as means \pm standard deviations (SD). Plasma concentration data were subjected to non-linear regression analysis using Drug and Statistics Software (DAS, version 2.0, Mathematical Pharmacology Professional Committee of China). Data from the MCAO study were subjected to analysis of variance using Excel 2010 and SPSS software (version 18.0). The $\mathrm{LD}_{50}$ and the associated $95 \%$ confidence interval of Nimotop injection were calculated using SPSS software (version 18.0) with the Bliss method. Differences between the different groups were determined using unpaired t-tests. $\mathrm{P}$ values $<0.05$ were considered statistically significant.

\section{Results and Discussion \\ Characterization of NIMO-TNE Drug Distribution in NIMO-TNE}

Drug distribution of NIMO-TNE is summarized in Table 1. The average drug content was $35.35 \% \pm 1.26 \%$ in the oil phase, $0.10 \% \pm 0.03 \%$ in the aqueous phase, and $64.56 \% \pm$ $1.26 \%$ in the phospholipid bilayer. The majority of NIMO in NIMO-TNE was dispersed in the phospholipids layer and the oil phase because NIMO is poorly water soluble.,19 
Table I Drug Distribution in NIMO-TNE at a Concentration of $1.0 \mathrm{mg} / \mathrm{mL}$

\begin{tabular}{|l|l|l|l|}
\hline \multirow{2}{*}{ Sample } & \multicolumn{3}{|l|}{ Drug Content (\%) } \\
\cline { 2 - 4 } & Oil Phase & Aqueous Phase & Phospholipid Bilayer \\
\hline NIMO-TNE & $35.35 \pm 1.26$ & $0.10 \pm 0.03$ & $64.56 \pm 1.26$ \\
\hline
\end{tabular}

Note: All results are represented as means $\pm S D(n=3)$.

Abbreviation: NIMO-TNE, two-vial formulation of NIMO-loaded nanoemulsion.

\section{Physical Stability Study}

Physical stability of NIMO-TNE and NIMO-PEG400 solution was evaluated, and the results are summarized in Table 2. The results showed that NIMO-TNE had an average particle size of $241.53 \mathrm{~nm}$, an average PDI of 0.11 , and a $\mathrm{pH}$ value of 7.23. These results were consistent throughout the experiments. Drug content of NIMO-TNE $(1.0 \mathrm{mg} / \mathrm{mL}$ drug load) remained stable for $24 \mathrm{~h}$. In the clinic, NIMO-TNE is prepared by mixing the blank fat emulsion and NIMO-PEG400 solution immediately prior to use. Therefore, $24 \mathrm{~h}$ stability was appropriate for clinical use. In addition, no significant changes were observed during long-term stability evaluation of NIMO-PEG400, as shown in Table 3. These results showed that NIMO-TNE was stable at $25{ }^{\circ} \mathrm{C} \pm 2{ }^{\circ} \mathrm{C}$ at a relative humidity of $60 \% \pm 5 \%$ for at least $24 \mathrm{~h}$, which is appropriate for clinical use.

Table 2 Physical Stability of NIMO-TNE (I.0 mg/mL)

\begin{tabular}{|l|l|l|l|l|}
\hline $\begin{array}{l}\text { Time } \\
\text { (h) }\end{array}$ & $\begin{array}{l}\text { Particle } \\
\text { Size (nm) }\end{array}$ & PDI & pH value & $\begin{array}{l}\text { Drug } \\
\text { Content (\%) }\end{array}$ \\
\hline 0 & $241.53 \pm 1.48$ & $0.11 \pm 0.02$ & $7.23 \pm 0.03$ & $100 *$ \\
2 & $239.10 \pm 1.12$ & $0.10 \pm 0.01$ & $7.20 \pm 0.04$ & $100.16 \pm 0.31$ \\
4 & $243.00 \pm 1.51$ & $0.10 \pm 0.01$ & $7.21 \pm 0.03$ & $100.72 \pm 0.49$ \\
6 & $243.07 \pm 1.22$ & $0.13 \pm 0.03$ & $7.19 \pm 0.04$ & $100.60 \pm 0.64$ \\
8 & $242.10 \pm 3.82$ & $0.11 \pm 0.02$ & $7.21 \pm 0.04$ & $99.75 \pm 0.59$ \\
10 & $244.13 \pm 2.58$ & $0.10 \pm 0.01$ & $7.21 \pm 0.03$ & $99.91 \pm 0.42$ \\
12 & $243.40 \pm 2.78$ & $0.12 \pm 0.01$ & $7.27 \pm 0.04$ & $99.23 \pm 0.37$ \\
24 & $245.40 \pm 2.42$ & $0.13 \pm 0.01$ & $7.26 \pm 0.04$ & $99.03 \pm 0.25$ \\
\hline
\end{tabular}

Notes: All results are presented as means \pm SD $(n=3)$. *The drug content of NIMO at $0 \mathrm{~h}$ was set as $100 \%$, and the drug content of NIMO at the remaining time points was expressed as the ratio of drug at each timepoint to that at $0 \mathrm{~h}$.

Abbreviations: NIMO-TNE, two-vial formulation of nimodipine-loaded nanoemulsion; PDI, polydispersity index.

\section{Dilution Stability Study}

Stability of diluted NIMO-TNE was evaluated because this formulation must be diluted prior to clinical use. When the emulsion was diluted to $0.04 \mathrm{mg} / \mathrm{mL}$, the concentration of Nimotop injection used clinically, no significant changes in particle size, PDI, $\mathrm{pH}$, or drug content were observed (Tables 4 and 5). The $\mathrm{pH}$ of diluted injection solutions increased slightly over time, but the changes were not statistically significant ( $\mathrm{p}>0.5$; Tables 4 and 5). A previous study showed that dilution of Nimotop injection resulted in precipitation of drug crystals due to the reduced ratio of organic solvent, which could result in increased risk to the patient. ${ }^{8}$ In contrast, the drug in NIMO-TNE was dissolved in the phospholipid layer and oil phase because nimodipine is highly lipophilic, which resulted in excellent stability following dilution. These results showed that NIMO-TNE could be diluted to $0.04 \mathrm{mg} / \mathrm{mL}$ and remain stable within $24 \mathrm{~h}$ at the same concentration of Nimotop injection used clinically.

\section{In vivo Pharmacokinetic Study}

Mean plasma concentration-time profiles following single intravenous injections of Nimotop and NIMO-TNE are shown in Figure 2. The results showed that Nimotop injection and NIMO-TNE had similar pharmacokinetic profiles. The drug was eliminated rapidly in the first $60 \mathrm{~min}$, after which elimination occurred more slowly. The drug was not detectable at $240 \mathrm{~min}$ for either Nimotop or NIMO-TNE. The main pharmacokinetic parameters of both groups are summarized in Table 6 . The results showed that the area under the plasma-time curve, mean residence time, and half-life were similar between the two groups $(\mathrm{p}>0.05)$, which indicated that Nimotop and NIMO-TNE were bioequivalent. The rapid elimination rate of NIMO-TNE may have been due to rapid release of drug from NIMO-TNE as a result of high drug content $(64.56 \% \pm 1.26 \%)$ in the phospholipid bilayer. ${ }^{8,21}$ In addition, a previous study showed that the plasma proteinbinding rate of nimodipine was $97-99 \%$ in blood. ${ }^{7}$ The released drug might interact with plasma proteins to promote further drug release. An MCAO model was used to evaluate

Table 3 Long-Term Stability of NIMO-PEG400

\begin{tabular}{|l|l|l|l|l|l|l|l|}
\hline Time (Month) & $\mathbf{0}$ & $\mathbf{I}$ & $\mathbf{2}$ & $\mathbf{3}$ & $\mathbf{6}$ & $\mathbf{9}$ & $\mathbf{1 2}$ \\
\hline Content (\%) & $100 *$ & $99.21 \pm 0.31$ & $100.53 \pm 0.36$ & $100.93 \pm 0.31$ & $99.95 \pm 0.62$ & $100.58 \pm 0.99$ & $100.20 \pm 0.85$ \\
\hline
\end{tabular}

Notes: All results are presented as means \pm SD $(n=3)$. *Drug content of NIMO at 0 h was set to $100 \%$, and drug content of NIMO at the remaining time points was expressed as the ratio of drug content at each time point versus the drug content at $0 \mathrm{~h}$.

Abbreviation: NIMO-PEG400, nimodipine-polyethylene glycol 400. 
Table 4 Physical Stability of NIMO-TNE Following Dilution to $0.04 \mathrm{mg} / \mathrm{mL}$ in $5 \%$ Glucose

\begin{tabular}{|l|l|l|l|l|}
\hline Time(h) & $\begin{array}{l}\text { Particle Size } \\
(\mathbf{n m})\end{array}$ & PDI & $\mathbf{p H}$ & Content (\%) \\
\hline 0 & $240.23 \pm 1.97$ & $0.09 \pm 0.03$ & $4.57 \pm 0.06$ & $100^{*}$ \\
2 & $238.17 \pm 0.84$ & $0.06 \pm 0.03$ & $4.62 \pm 0.03$ & $100.33 \pm 0.37$ \\
4 & $239.80 \pm 2.79$ & $0.09 \pm 0.02$ & $4.61 \pm 0.05$ & $99.91 \pm 0.16$ \\
6 & $237.63 \pm 3.48$ & $0.08 \pm 0.02$ & $4.62 \pm 0.04$ & $100.81 \pm 1.04$ \\
8 & $239.73 \pm 2.54$ & $0.10 \pm 0.02$ & $4.65 \pm 0.04$ & $100.49 \pm 0.40$ \\
10 & $237.23 \pm 2.96$ & $0.08 \pm 0.02$ & $4.70 \pm 0.01$ & $100.30 \pm 0.74$ \\
12 & $236.10 \pm 1.77$ & $0.09 \pm 0.03$ & $4.71 \pm 0.05$ & $99.36 \pm 0.76$ \\
24 & $238.93 \pm 3.18$ & $0.08 \pm 0.02$ & $4.83 \pm 0.04$ & $99.28 \pm 0.16$ \\
\hline
\end{tabular}

Notes: All the results are represented as mean $\pm S D(n=3)$. *Drug content at 0 $\mathrm{h}$ was set to $100 \%$ and NIMO content at the remaining time points was expressed as the ratio of drug content at each time point to drug content at $0 \mathrm{~h}$.

Abbreviations: NIMO-TNE, two-vial formulation of nimodipine-loaded nanoemulsion; PDI, polydispersity index.

Table 5 Physical Stability of NIMO-TNE Following Dilution to $0.04 \mathrm{mg} / \mathrm{mL}$ in $0.9 \%$ Sodium Chloride

\begin{tabular}{|l|l|l|l|l|}
\hline Time (h) & $\begin{array}{l}\text { Particle Size } \\
(\mathbf{n m})\end{array}$ & PDI & $\mathbf{p H}$ & Content (\%) \\
\hline 0 & $227.50 \pm 1.63$ & $0.05 \pm 0.03$ & $5.89 \pm 0.08$ & $100^{*}$ \\
2 & $227.10 \pm 1.04$ & $0.06 \pm 0.03$ & $5.92 \pm 0.04$ & $100.74 \pm 0.48$ \\
4 & $232.27 \pm 3.64$ & $0.06 \pm 0.04$ & $5.96 \pm 0.05$ & $99.33 \pm 0.41$ \\
6 & $228.60 \pm 3.72$ & $0.06 \pm 0.03$ & $5.94 \pm 0.04$ & $100.53 \pm 0.06$ \\
8 & $232.80 \pm 1.14$ & $0.04 \pm 0.01$ & $5.95 \pm 0.03$ & $99.31 \pm 0.74$ \\
10 & $231.00 \pm 0.99$ & $0.05 \pm 0.02$ & $6.01 \pm 0.03$ & $100.54 \pm 0.89$ \\
12 & $231.73 \pm 1.61$ & $0.05 \pm 0.02$ & $5.96 \pm 0.01$ & $99.36 \pm 0.76$ \\
24 & $233.00 \pm 3.28$ & $0.03 \pm 0.02$ & $5.96 \pm 0.03$ & $97.34 \pm 0.84$ \\
\hline
\end{tabular}

Notes: All the results are represented as mean $\pm S D(n=3)$. *Drug content at 0 $\mathrm{h}$ was set to $100 \%$ and NIMO content at the remaining time points was expressed as the ratio of drug content at each time point to drug content at $0 \mathrm{~h}$.

Abbreviations: NIMO-TNE, two-vial formulation of nimodipine-loaded nanoemulsion; PDI, polydispersity index.

the potential for treatment of ischemic cerebrovascular disease using NIMO-TNE.

\section{In vivo Pharmacodynamic Study}

No deaths occurred during the experimental procedures. The infarction areas in the Nimotop injection group and the NIMO-TNE group were significantly smaller than those in the normal saline group, and no significant differences in infarct size were observed between the NIMO-TNE injection group and the Nimotop injection group (Figure 3A). These results were consistent with the quantitative results summarized in Figure 3B, in which the average rate of infarctionin the NS group was $37.70 \%$ $\pm 4.08 \%$, the half-cerebral infarction ratio in the Nimotop injection group was $17.34 \% \pm 3.24 \%$, and the halfcerebral infarction ratio in the NIMO-TNE group was

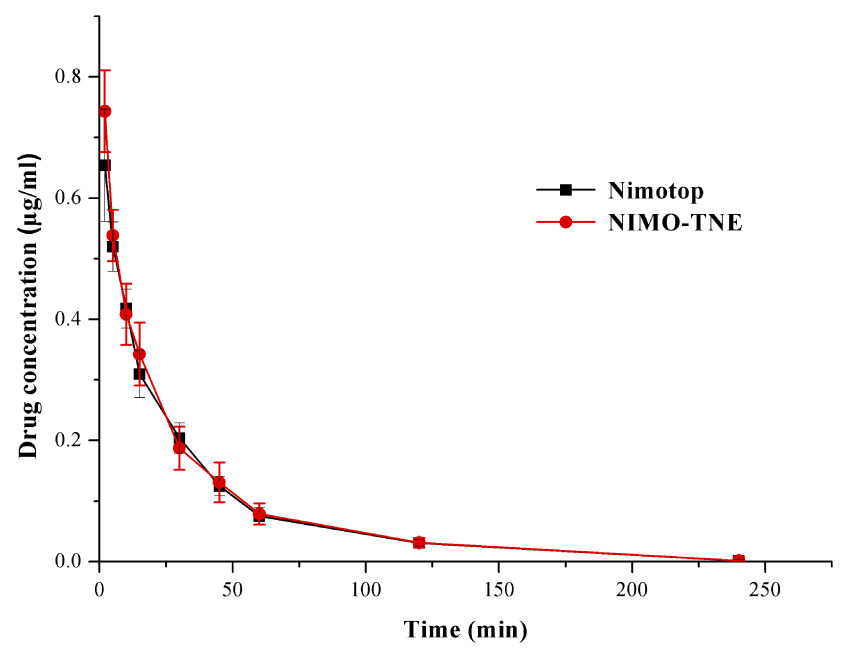

Figure 2 Mean plasma concentration-time profiles following a single intravenous injection of Nimotop or NIMO-TNE in rats $(n=6)$.

Abbreviation: NIMO-TNE, two-vial formulation of nimodipine-loaded nanoemulsion.

$17.51 \% \pm 3.42 \%$. The reduction in rate of half-cerebral infarction in response to Nimotop injection and NIMOTNE compared to the NS group was 54.03\% $\pm 8.60 \%$ and $53.56 \% \pm 9.08 \%$, respectively (Figure 3C). There was no difference in the rate of reduction of halfcerebral infarction between the Nimotop injection group and the NIMO-TNE group $(\mathrm{p}>0.05)$. These results indicated that a single intravenous injection of NIMOTNE significantly reduced infarct size in a rat MCAO model. Furthermore, NIMO-TNE reduced infarct size to a similar degree as Nimotop injection at the same dose.

\section{Intravenous Injection Safety Evaluation of Hemolysis}

The results of the hemolysis test were shown in Figure 4. Complete hemolysis was observed in the positive control tube (No. 7) at $0 \mathrm{~min}$, as evidenced by a clear red solution with no erythrocytes at the bottom of the tube. ${ }^{33,39}$ In

Table 6 Pharmacokinetic Parameters of Nimotop Injection and NIMO-TNE in Rats

\begin{tabular}{|l|l|l|l|}
\hline Parameters & Unit & Nimotop & NIMO-TNE \\
\hline AUC $(0-\mathrm{t})$ & $\mathrm{mg} / \mathrm{L} \cdot \mathrm{min}$ & $20.208 \pm \mathrm{I} .514$ & $21.03 \mathrm{I} \pm 3.179$ \\
AUC $(0-\infty)$ & $\mathrm{mg} / \mathrm{L} \cdot \mathrm{min}$ & $20.296 \pm 1.577$ & $21.120 \pm 3.262$ \\
MRT $(0-\mathrm{t})$ & $\mathrm{min}$ & $38.767 \pm 3.170$ & $38.795 \pm 2.760$ \\
MRT $(0-\infty)$ & $\mathrm{min}$ & $39.814 \pm 3.246$ & $40.702 \pm 4.433$ \\
$\mathrm{t}_{\mathrm{I} / 2 \mathrm{z}}$ & $\mathrm{min}$ & $30.753 \pm 4.527$ & $30.299 \pm 4.959$ \\
$\mathrm{CLz}$ & $\mathrm{L} / \mathrm{min} / \mathrm{kg}$ & $0.040 \pm 0.003$ & $0.039 \pm 0.006$ \\
\hline
\end{tabular}

Note: All results are presented as means $\pm S D(n=6)$.

Abbreviations: NIMO-TNE, two-vial formulation of nimodipine-loaded nanoemulsion; AUC, area under the plasma level time curve; MRT, mean residence time. 
A
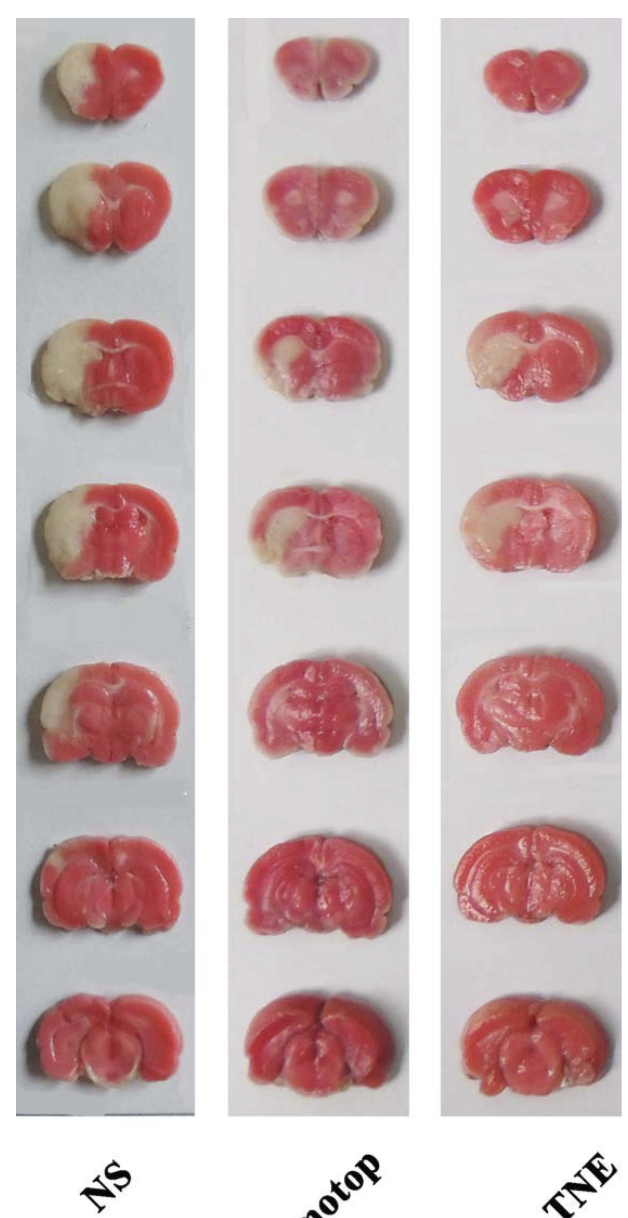
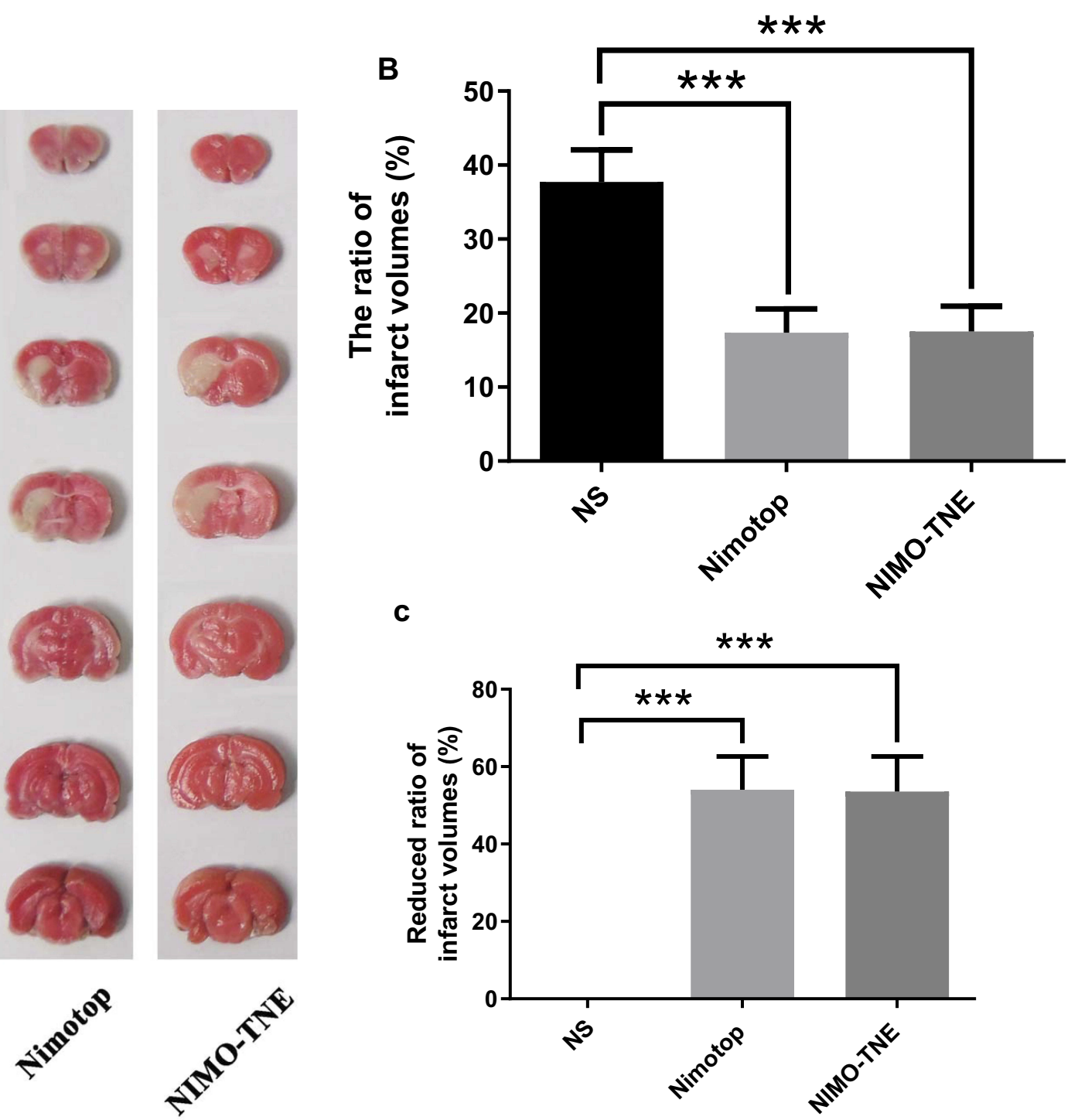

C

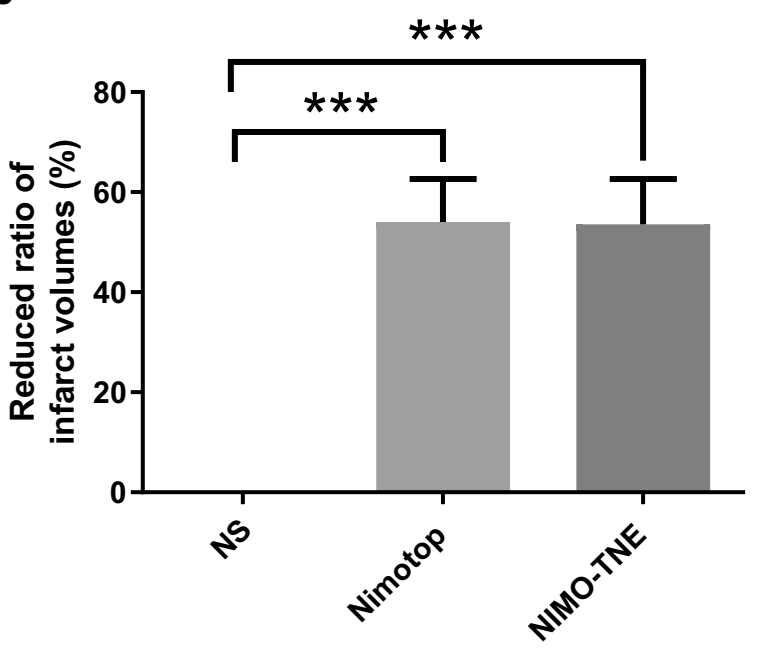

Figure 3 Brain infarction in a rat MCAO model. (A) Representative photographs of TTC stained brain slices from different groups. (B). The ratio of infarct volumes following MCAO in rats. (C) Reduced ratio of infarct volumes following Nimotop injection or NIMO-TNE injection versus the NS group.

Notes: Normal saline (NS) group $(n=8)$; Nimotop injection group $(n=8)$; NIMO-TNE group $(n=8)$. ***p $<0.001$ vs NS group.

Abbreviations: NS, Normal saline; NIMO-TNE, two-vial formulation of nimodipine-loaded nanoemulsion; MCAO, Middle cerebral artery occlusion.

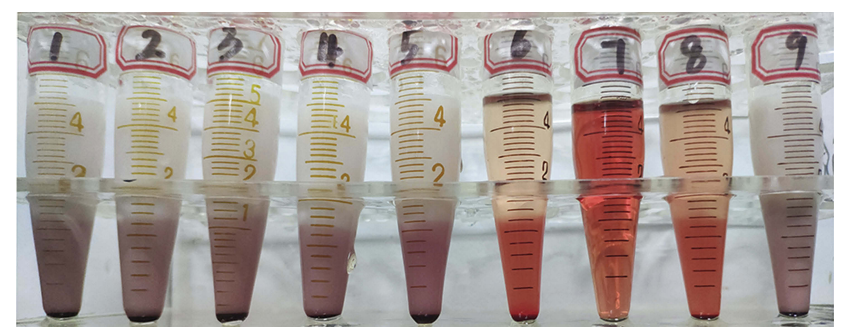

Figure 4 Hemolysis results for NIMO-TNE at $4 \mathrm{~h}$.

Abbreviation: NIMO-TNE, two-vial formulation of nimodipine-loaded nanoemulsion.

contrast, no hemolysis was observed in the negative control (No. 6) or in the Nimotop injection tube (No. 8), and erythrocytes precipitated at the bottom of each tube. The
NIMO-TNE (No. 1-5) and BNE (No.9) tubes were divided into three layers, including an emulsion layer, a mixed emulsion and erythrocyte layer, and an erythrocyte layer. The emulsion layer thickened over time, and the layer containing emulsion and erythrocytes thinned over time, and erythrocytes precipitated at the bottoms of the tubes. These results indicated that NIMO-TNE $(1 \mathrm{mg} /$ $\mathrm{mL}$ ) did not induce hemolysis at $37^{\circ} \mathrm{C}$.

\section{Intravenous Irritation Assessment}

The intravenous irritation test was performed to compare irritation resulting from saline injection and NIMO-TNE to that resulting from Nimotop injection. Mice in the 
Nimotop group experienced more irritation than those in the NS or NIMO-TNE groups during administration. In addition, Nimotop injection induced edema, which indicated severe irritation during administration. ${ }^{40}$ In contrast, no significant changes were observed in the ears of rabbits in the NS or NIMO-TNE groups following infusion.

Images of the rabbit ear tissue sections from the injection site are shown in Figure 5. The rabbit ear marginal vein in the Nimotop injection group showed vasodilation, hemorrhage, and edema around the vein. In addition, these tissue sections showed inflammatory cell infiltration in the vessel wall and surrounding tissues, and endothelial cell swelling, which indicated severe vascular irritation. In contrast, no inflammatory cell infiltration was observed in the surrounding tissues in the NS or NIMO-TNE groups, which indicated that no irritation had occurred. These differences may have been due to high ethanol content in Nimotop injection. The high concentration of ethanol may have induced vasodilation and increased vascular permeability, which results in leakage of red blood cells, drug, and organic solvent. ${ }^{8,40}$ These results indicated that Nimotop injection may induce significant pain and serious vascular irritation. The results showed that NIMOTNE did not induce adverse effects such as pain and vascular irritation, which represented a marked improvement compared to Nimotop injection.
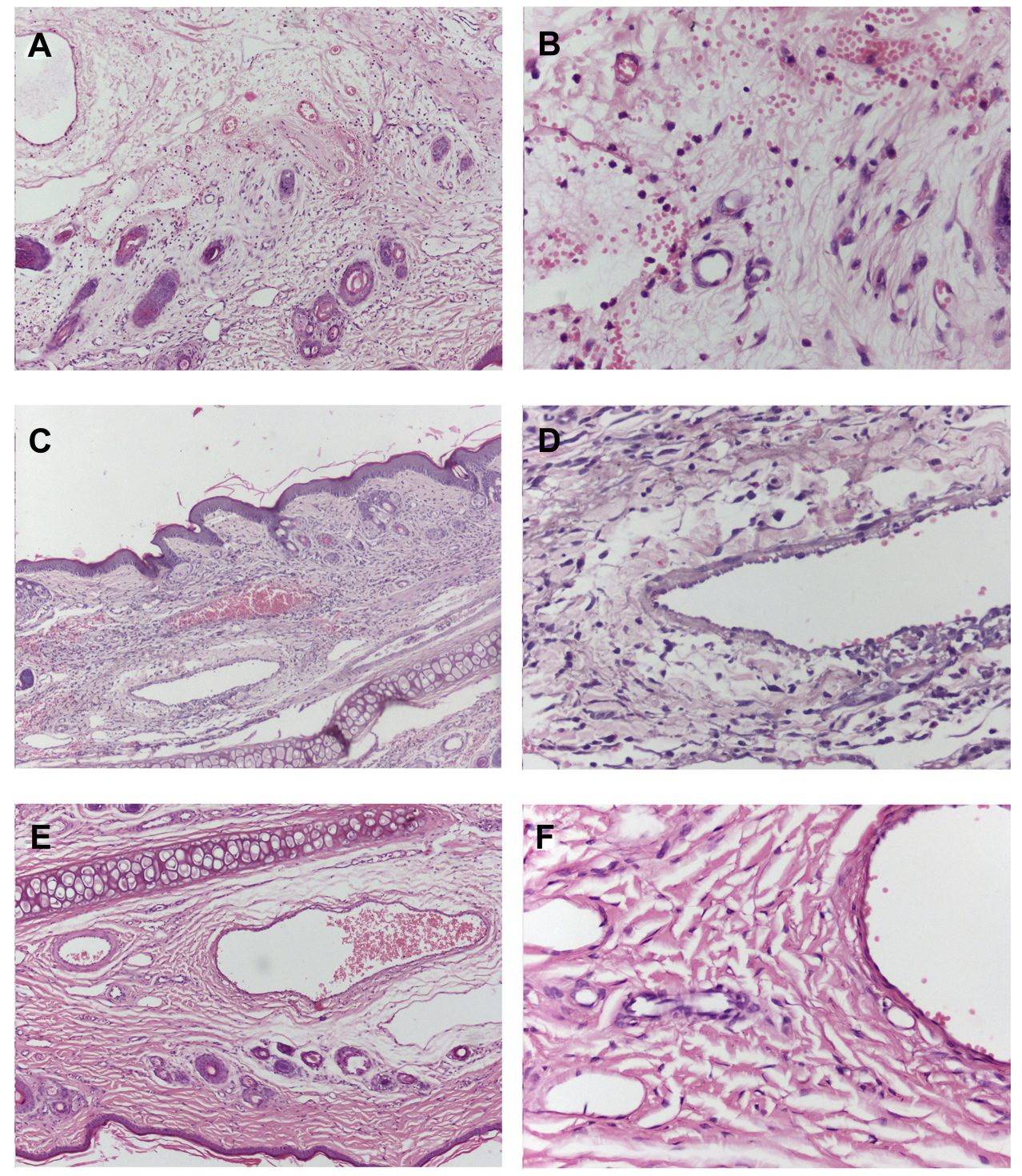

Figure 5 Images of rabbit ear pathological sections. (A) Nimotop injection group (I00x); (B) Nimotop injection group (400x); (C) NIMO-TNE group (I00x); (D) NIMOTNE group (400x); (E) NS group (100x); (F) NS group (400x).

Abbreviations: NIMO-TNE, two-vial formulation of nimodipine-loaded nanoemulsion; NS, Normal saline. 


\section{Acute Toxicity}

As shown in Table 7, acute toxicity of Nimotop injection increased in a dose-dependent manner from 1.90 to $2.83 \mathrm{mg} /$ $\mathrm{kg}$. The clinical signs of toxicity were asthenia, spasticity of the hind limbs, and tics. In addition, urinary incontinence was observed in mice that died as a result of the injection. All the surviving mice recovered after 14 days. The $\mathrm{LD}_{50}$ of Nimotop injection was estimated to be $2.35 \mathrm{mg} / \mathrm{kg}$, and the $95 \%$ confidence interval was $2.20-2.51 \mathrm{mg} / \mathrm{kg}$.

In the maximum feasible dose (MFD) test of NIMOTNE, no abnormal behavior or obvious signs of toxicity were observed following injection. Changes in body weight were similar between control mice and those treated with NIMO-TNE (Figure 6). The MFD of NIMO-TNE was at least $54 \mathrm{mg} / \mathrm{kg}$.

Given that the $\mathrm{LD}_{50}$ of Nimotop injection was $2.35 \mathrm{mg} / \mathrm{kg}$, and NIMO-TNE could be dosed at $54 \mathrm{mg} / \mathrm{kg}$ with no obvious toxicity or side effects, we concluded that NIMO-TNE was significantly safer than Nimotop injection. These results were consistent with those from the intravenous irritation test.

\section{Conclusion}

In summary, NIMO-TNE was successfully prepared using a simple method. The drug loading of NIMO-TNE reached $1 \mathrm{mg} / \mathrm{mL}$, and $64.56 \% \pm 1.26 \%$ and $35.35 \% \pm 1.26 \%$ of the drug was distributed in the phospholipid bilayer and oil phase, respectively. NIMO-TNE was stable for a sufficient duration to allow for clinical application. In addition, NIMO-TNE was physically compatible with $5 \%$ glucose and $0.9 \%$ sodium chloride, as indicated by stability for 24 $\mathrm{h}$ in these solutions. NIMO-TNE showed similar pharmacokinetic properties to Nimotop injection. Moreover, there were no significant differences in reduction of MCAOinduced brain ischemia following NIMO-TNE or Nimotop injection in rats. The hemolysis test showed that NIMO-TNE did not induce hemolysis at $37{ }^{\circ} \mathrm{C}$. Furthermore, NIMO-TNE did not induce irritation during or after intravenous infusion, which represented a significant improvement over Nimotop injection. In

Table 7 Acute Toxicity of Nimotop Injection in Mice

\begin{tabular}{|l|l|l|}
\hline Dose (mg/kg) & Mortality & Mortality (\%) \\
\hline 1.90 & $0 / 8$ & 0.0 \\
2.10 & $2 / 8$ & 25.0 \\
2.32 & $4 / 8$ & 50.0 \\
2.56 & $5 / 8$ & 62.5 \\
2.83 & $8 / 8$ & 100.0 \\
\hline
\end{tabular}

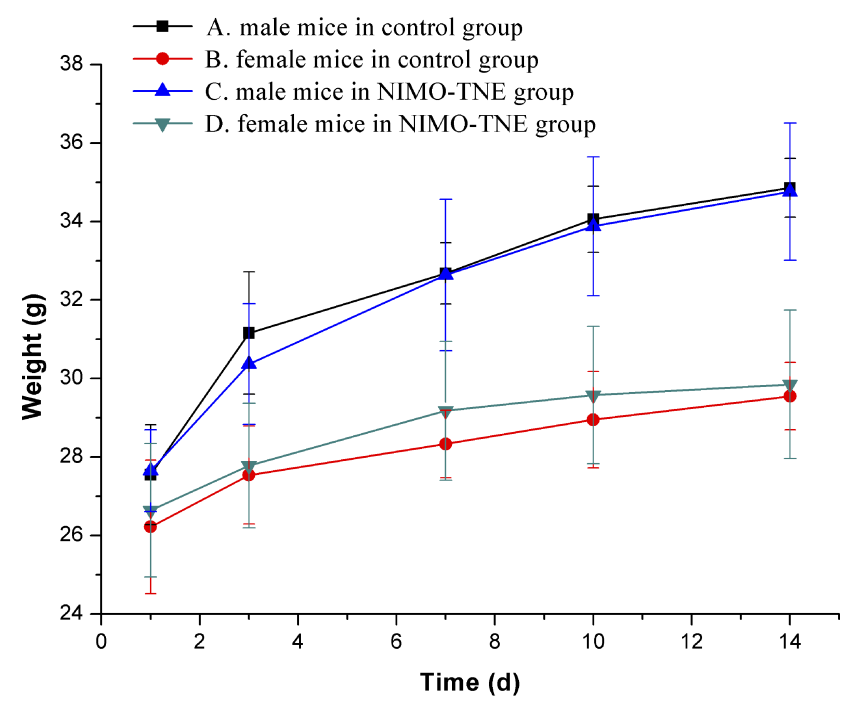

Figure 6 Weight change curve. A. Male mice in the control group $(n=6)$. B. Female mice in the control group $(n=6)$. C. Male mice in the NIMO-TNE group $(n=6)$. D. Female mice in the NIMO-TNE group $(n=6)$.

Abbreviation: NIMO-TNE, two-vial formulation of nimodipine-loaded nanoemulsion.

addition, NIMO-TNE could be dosed at $54 \mathrm{mg} / \mathrm{kg}$ with no obvious toxicity or side effects, whereas the $\mathrm{LD}_{50}$ of Nimotop injection was $2.35 \mathrm{mg} / \mathrm{kg}$. Therefore, NIMOTNE may be a promising drug carrier for NIMO. Furthermore, this novel two-vial formulation of a drugloaded nanoemulsion may be a promising method for the intravenous administration of lipophilic drugs.

\section{Abbreviations}

NIMO, Nimodipine; NIMO-TNE, two-vial formulation of nimodipine-loaded nanoemulsion; PEG400, Polyethylene glycol 400; NIMO-PEG400, Nimodipine-polyethylene glycol 400; BNE, Blank nanoemulsion; DL, Drug loading; HPLC, High performance liquid chromatography; PDI, Polydispersity index; NS, Normal saline; MCAO, Middle cerebral artery occlusion; LD50, Median lethal dose; MFD, Maximal feasible dose; SD, Standard deviation; AUC, Area under the plasma level time curve; MRT, Mean residence time.

\section{Acknowledgments}

This study was supported by Shanghai Key Laboratory of New Drug Design (Grant No. 17DZ2271000), the National Natural Science Foundation of China (NO. 81772749), Shanghai Rising-Star Program (NO. 18QB1400400), Shanghai Qingpu District Industry-University-Research Cooperation Development Fund Project (QIUR 2019-5), and Shanghai Science and Technology Project of Little Giant (1902HX76600). 


\section{Disclosure}

The authors report no conflicts of interest in this work.

\section{References}

1. Zhao Y, Xin T, Ye T, Yang X, Pan W. Solid dispersion in the development of a nimodipine delayed-release tablet formulation. Asian J Pharm Sci. 2014;9(1):35-41. doi:10.1016/j.ajps.2013.11.006

2. Barmpalexis P, Kanaze FI, Kachrimanis K, Georgarakis E. Artificial neural networks in the optimization of a nimodipine controlled release tablet formulation. Eur $J$ Pharm Biopharm. 2010;74 (2):316-323. doi:10.1016/j.ejpb.2009.09.011

3. Gelmers HJ. Calcium-channel blockers in the treatment of migraine. Am J Cardiol. 1985;55:3. doi:10.1016/0002-9149(85)90622-8

4. Caceres JF, Blangy D, Glikin GC. Requirement of DNA topoisomerases for in vitro chromatin assembly by 3 T6 mouse cell extracts. Eur J Biochem. 1989;181(2):531-537. doi:10.1111/ejb.1989.181.issue-2

5. Sun Y, Rui Y, Wenliang Z, Tang X. Nimodipine semi-solid capsules containing solid dispersion for improving dissolution. Int J Pharm. 2008;359(1-2):144-149. doi:10.1016/j.ijpharm.2008.03.040

6. Soliman GM, Sharma R, Choi AO, et al. Tailoring the efficacy of nimodipine drug delivery using nanocarriers based on A 2 B miktoarm star polymers. Biomaterials. 2010;31(32):8382-8392. doi:10.1016/j.biomaterials.2010.07.039

7. Hu FQ, Zhang Y, Du YZ, Yuan H. Nimodipine loaded lipid nanospheres prepared by solvent diffusion method in a drug saturated aqueous system. Int J Pharm. 2008;348(1-2):146-152. doi:10.1016/ j.ijpharm.2007.07.025

8. Song X, Jiang Y, Ren C, et al. Nimodipine-loaded mixed micelles: formulation, compatibility, pharmacokinetics, and vascular irritability study. Int J Nanomedicine. 2012;7:3689-3699. doi:10.2147/IJN.S33228

9. Li J, Fan L, Yuan M, Xing M. Salidroside inhibits lipopolysaccharide-ethanol-induced activation of proinflammatory macrophages via notch signaling pathway. Curr Med Sci. 2019;39 (4):526-533. doi:10.1007/s11596-019-2069-4

10. Shirpoor A, Gharalari FH, Rasmi Y, Heshmati E. Ginger extract attenuates ethanol-induced pulmonary histological changes and oxidative stress in rats. $J$ Biomed Res. 2017;31(6):521-527.

11. Le Daré B, Victoni T, Bodin A, et al. Ethanol upregulates the P2X7 purinergic receptor in human macrophages. Fundam Clin Pharmacol. 2019;33(1):63-74. doi:10.1111/fcp.2019.33.issue-1

12. Schneider ACR, Gregório C, Uribe-Cruz C, et al. Chronic exposure to ethanol causes steatosis and inflammation in zebrafish liver. World J Hepatol. 2017;9(8):418-426. doi:10.4254/wjh.v9.i8.418

13. Zamani E, Mohammadbagheri M, Fallah M, Shaki F. Atorvastatin attenuates ethanol-induced hepatotoxicity via antioxidant and anti-inflammatory mechanisms. Res Pharm Sci. 2017;12 (4):315-321. doi:10.4103/1735-5362.212049

14. Garg NK, Tyagi RK, Singh B, et al. Nanostructured lipid carrier mediates effective delivery of methotrexate to induce apoptosis of rheumatoid arthritis via NF- $\mathrm{KB}$ and FOXO1. Int J Pharm. 2016;499 (1-2):301-320. doi:10.1016/j.ijpharm.2015.12.061

15. Rashed HM, Shamma RN, Basalious EB. Contribution of both olfactory and systemic pathways for brain targeting of nimodipine-loaded lipo-pluronics micelles: in vitro characterization and in vivo biodistribution study after intranasal and intravenous delivery. Drug Deliv. 2017;24(1):181-187. doi:10.1080/10717544.2016.1236848

16. Ji B, Wang M, Gao D, et al. Combining nanoscale magnetic nimodipine liposomes with magnetic resonance image for Parkinson's disease targeting therapy. Nanomedicine-UK. 2017;12(3):237-253. doi:10.2217/nnm-2016-0267

17. Moreno LCGEAI, Cavalcanti IMF, Satyal P, Santos-Magalhães NS, Rolim HML, Freitas RM. Acute toxicity and anticonvulsant activity of liposomes containing nimodipine on pilocarpine-induced seizures in mice. Neurosci Lett. 2015;585:38-42. doi:10.1016/j.neulet.2014.11.025
18. Xiong R, Lu W, Li J, Wang P, Xu R, Chen T. Preparation and characterization of intravenously injectable nimodipine nanosuspension. Int J Pharm. 2008;350(1-2):338-343. doi:10.1016/ j.ijpharm.2007.08.036

19. Ding Y, Teng Z, Zhou J, et al. Preparation and characterization of nimodipine-loaded nanostructured lipid systems for enhanced solubility and bioavailability. Int $J$ Nanomedicine. 2018;14:119-133. doi:10.2147/IJN.S186899

20. Guada M, Lasa-Saracíbar B, Lana H, Del Carmen Dios-Viéitez M, Blanco-Prieto MJ. Lipid nanoparticles enhance the absorption of cyclosporine A through the gastrointestinal barrier: in vitro and in vivo studies. Int $J$ Pharm. 2016;500(1-2):154-161. doi:10.1016/ j.ijpharm.2016.01.037

21. Jing X, Deng L, Gao B, et al. A novel polyethylene glycol mediated lipid nanoemulsion as drug delivery carrier for paclitaxel. Nanomed Nanotechnol Biol Med. 2014;10(2):371-380. doi:10.1016/j. nano.2013.07.018

22. Chen L, Chen B, Deng L, et al. An optimized two-vial formulation lipid nanoemulsion of paclitaxel for targeted delivery to tumor. Int J Pharm. 2017;534(1-2):308-315. doi:10.1016/j.ijpharm.2017.10. 005

23. Chalikwar SS, Belgamwar VS, Talele VR, Surana SJ, Patil MU. Formulation and evaluation of nimodipine-loaded solid lipid nanoparticles delivered via lymphatic transport system. Colloid Surface B. 2012;97:109-116. doi:10.1016/j.colsurfb.2012.04.027

24. Guan T, Miao Y, Xu L, et al. Injectable nimodipine-loaded nanoliposomes: preparation, lyophilization and characteristics. Int J Pharm. 2011;410(1-2):180-187. doi:10.1016/j.ijpharm.2011. 03.009

25. Tian S, Gao W, Liu Y, Kang W. Study on the stability of heavy crude oil-in-water emulsions stabilized by two different hydrophobic amphiphilic polymers. Colloids Surfaces a Physicochem Eng Asp. 2019;572:299-306. doi:10.1016/j.colsurfa.2019.04.017

26. Sun C, Wang J, Liu J, Qiu L, Zhang W, Zhang L. Liquid proliposomes of nimodipine drug delivery system: preparation, characterization, and pharmacokinetics. AAPS PharmSciTech. 2013;14(1):332-338. doi:10.1208/s12249-013-9924-6

27. Liu DZ, Jickling GC, Ander BP, et al. Elevating microRNA-122 in blood improves outcomes after temporary middle cerebral artery occlusion in rats. $J$ Cereb Blood Flow Metab. 2016;36 (8):1374-1383. doi:10.1177/0271678X15610786

28. Tamura A, Graham DI, McCulloch J, Teasdale GM. Focal cerebral ischaemia in the rat: I. Description of technique and early neuropathological consequences following middle cerebral artery occlusion. J Cereb Blood Flow Metab. 1981;1(1):53-60. doi:10. 1038/jcbfm. 1981.6

29. Bederson JB, Pitts LH, Tsuji M, Nishimura MC, Davis RL, Bartkowski $\mathrm{H}$. Rat middle cerebral artery occlusion: evaluation of the model and development of a neurologic examination. J Am Heart Assoc. 1986;17(3):472-476.

30. Xie HH, Zhang XF, Chen YY, Shen FM, Su DF. Synergism of hydrochlorothiazide and nifedipine on blood pressure variability reduction and organ protection in spontaneously hypertensive rats. Hypertens Res. 2008;31(4):685-691. doi:10.1291/hypres.31.685

31. Yu J, Li X, Matei N, et al. Ezetimibe, a NPC1L1 inhibitor, attenuates neuronal apoptosis through AMPK dependent autophagy activation after MCAO in rats. Exp Neurol. 2018;307(5):12-23. doi:10.1016/j. expneurol.2018.05.022

32. Wu JY, Li M, Cao LJ, et al. Protease Omi cleaving Hax-1 protein contributes to $\mathrm{OGD} / \mathrm{R}$-induced mitochondrial damage in neuroblastoma N2a cells and cerebral injury in MCAO mice. Acta Pharmacol Sin. 2015;36(9):1043-1052. doi:10.1038/aps.2015.50

33. Li L, Li W, Sun J, et al. Preparation and evaluation of progesterone nanocrystals to decrease muscle irritation and improve bioavailability. AAPS PharmSciTech. 2018;19(3):1254-1263. doi:10. 1208/s12249-017-0938-3 
34. Yang F, Yu XH, Qiao F, et al. Formulation and characterization of Brucea javanica oil microemulsion for improving safety. Drug Dev Ind Pharm. 2014;40(2):266-277. doi:10.3109/03639045.2012.756887

35. Harpur ES, Worah D, Hals PA, Holtz E, Furuhama K, Nomura H. Preclinical safety assessment and pharmacokinetics of gadodiamide injection, a new magnetic resonance imaging contrast agent. Invest Radiol. 1993;28:S28-S43. doi:10.1097/00004424199303001-00004

36. Torii Y, Goto Y, Nakahira S, Kozaki S, Kaji R, Ginnaga A. Comparison of systemic toxicity between botulinum toxin subtypes A1 and A2 in mice and rats. Basic Clin Pharmacol Toxicol. 2015;116 (6):524-528. doi:10.1111/bcpt.2015.116.issue-6

37. Misik J, Pavlikova R, Cabal J, Kuca K. Acute toxicity of some nerve agents and pesticides in rats. Drug Chem Toxicol. 2015;38(1):32-36. doi: $10.3109 / 01480545.2014 .900070$
38. Zhang Q, Li J, Zhang W, et al. Acute and sub-chronic toxicity studies of honokiol microemulsion. Regul Toxicol Pharmacol. 2015;71 (3):428-436. doi:10.1016/j.yrtph.2014.11.007

39. Li G, Zhao M, Zhao L. Well-defined hydroxyethyl starch-10-hydroxy camptothecin super macromolecule conjugate: cytotoxicity, pharmacodynamics research, tissue distribution test and intravenous injection safety assessment. Drug Deliv. 2016;23(8):2860-2868. doi:10.3109/ 10717544.2015.1110844

40. Lazarewicz JW, Pluta R, Saliriska E, Puka M. Beneficial effect of nimodipine on metabolic and functional disturbances in rabbit hippocampus following complete cerebral ischemia. Stroke. 1989;20 (1):70-77. doi:10.1161/01.STR.20.1.70

\section{Publish your work in this journal}

The International Journal of Nanomedicine is an international, peerreviewed journal focusing on the application of nanotechnology in diagnostics, therapeutics, and drug delivery systems throughout the biomedical field. This journal is indexed on PubMed Central, MedLine, CAS, SciSearch ${ }^{\mathbb{}}$, Current Contents ${ }^{\mathbb{R}} /$ Clinical Medicine,
Journal Citation Reports/Science Edition, EMBase, Scopus and the Elsevier Bibliographic databases. The manuscript management system is completely online and includes a very quick and fair peer-review system, which is all easy to use. Visit http://www.dovepress.com/ testimonials.php to read real quotes from published authors. 